\title{
Objective determination of human visual acuity from the visual evoked potential
}

\author{
VERNON L. TOWLE and M. RUSSELL HARTER \\ University of North Carolina, Greensboro, North Carolina 27412
}

\begin{abstract}
The natural visual acuities of 11 subjects were predicted from their flashed pattern evoked potentials. An objective data analysis technique was employed, based on the slope of the high spatial frequency side of the function between pattern element size and the amplitude of the evoked potential.
\end{abstract}

The inverted U-shaped function between the size of the elements in a patterned stimulus and the amplitude of the visual evoked potential (VEP) elicited by the stimulus (Harter \& White, 1970; Reitveld, Tordoir, Hagenouw, Lubbers, \& Spoor, 1967; and others) can be used as an index of visual functioning. Visual acuity has been assumed to be related to three aspects of this function (see Figure 1): (1) determining the point at which the function peaks (Harter \& Suitt, 1970; Harter \& White, 1970; Sokol \& Dobson, 1976), (2) determining the smallest pattern element for which a reliable pattern VEP can be obtained (Freeman \& Marg, 1975; Grall, Rigaudiere, Delthil, Legargasson, \& Sourdille, 1976; Harter, Deaton, \& Odom, 1977a; Marg, Freeman, Peltzman, \& Goldstein, 1976; Towle \& Harter, 1977), and (3) extrapolating the function to its high spatial frequency cutoff, or threshold (Berkley \& Watkins, 1973; Campbell, Maffei, \& Piccolino, 1973; Grall, Sourdille, Delthil, \& Ninnin, 1973; Harter, Deaton, \& Odom, 1977b). Only two of these studies have validated the VEP acuity estimates by systematically comparing the smallest pattern element that could be discriminated by psychophysical and VEP methods (Grall et al., 1976; Towle \& Harter, 1977).

In the present study, perceptual acuity was predicted on the basis of the slope of the high spatial frequency side of the function (4 in Figure 1). In addition to employing a different technique, this study differs from our earlier one (Towle \& Harter, 1977 ) in that monocular, rather than binocular, viewing conditions were used, and checkerboards, rather than dots, were used as the eliciting stimuli.

\section{METHOD}

\section{Subjects}

The authors and nine student volunteers with natural visual

This study was supported in part by a Sloan Foundation grant. V. L. Towle's present address: Department of Psychology, University of Illinois, Champaign, Illinois 61820 . Reprints may be obtained from the second author. acuities ranging from $2.2(20 / 9)$ to $.33(20 / 67)$ served as subjects. Four of them had participated in previous experiments in the laboratory.

\section{Visual Acuity}

Natural visual acuities were measured monocularly (right eye) with a Jaeger recognition acuity test. The Jaeger card was used because it could be observed at the same viewing distance employed to obtain evoked potentials $(81 \mathrm{~cm})$. The Jaeger measure of perceptual acuity was expressed in decimal units ${ }^{1}$ since this expands the high visual acuity end of the scale and resulted in a better linear relationship (a higher correlation) between changes in VEP amplitude as a function of log check size and perceptual acuity.

\section{Visual Stimuli}

VEPs were elicited by a diffuse light flash and a series of $B / W$ checkerboard flashes with individual check sizes subtending 6,8 , $12,16,24,32$, and $64 \mathrm{~min}$ of arc. These check sizes were selected since they provided a sufficient number of points along the high spatial frequency side of the evoked-potential size-amplitude function to determine the slope of this portion of the function. The eight stimulus transparencies were back-illuminated for $10 \mu \mathrm{sec}$

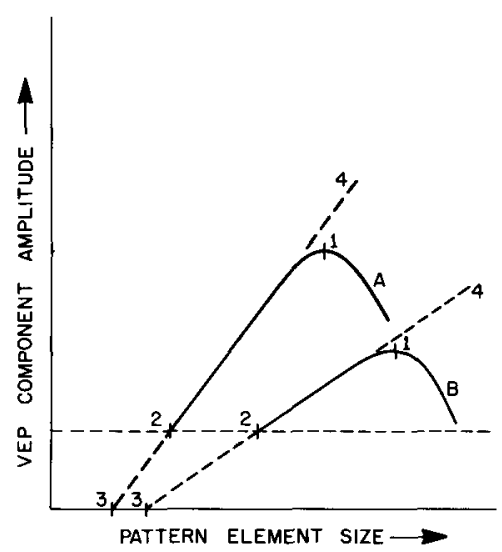

Figure 1. Schematized VEP size-amplitude functions indicating relatively good (A) and relatively poor (B) visual acuity. Four strategies may be used to predict visual acuity from changes in the evoked potential size-amplitude function: (1) the pattern size at which the function peaks; the observed pattern threshold (2) measured directly, or (3) extrapolated to the zero-voltage point; and (4) the slope of the ascending portion of the function. The horizontal dashed line represents the noise level of the VEP. 
with a Grass PS-2 photostimulator once every $600 \mathrm{msec}$. The space-average luminance of all stimulus flashes was $4.2 \log$ units above threshold. These flashes momentarily appeared in a relatively dark window $(1.8 \mathrm{~mL})$ surrounded by a constantly illuminated white field $(25.0 \mathrm{~mL})$. The display was $81 \mathrm{~cm}$ in front of the subject and subtended $4.3^{\circ} \times 5.9^{\circ}(61 \times 83 \mathrm{~mm})$. A laboratorybuilt high-speed random-access stimulator was used to flash the different stimuli (Musso \& Harter, 1975; Towle \& Harter, 1977).

\section{Procedure}

Subjects viewed the eight different stimuli with the right eye. The eight stimuli were presented in a quasi-random order (AAADDDGGGBBBEEEHHHCCCFFF) until each had been presented at least 64 times. The order of presentation was reversed for two of the four replications of each condition. The subjects were instructed to fixate a point $1 \mathrm{~cm}$ below the top center of the display and to stop the stimulus train with a button if they had difficulty maintaining fixation or accommodation.

\section{Average Evoked Potentials}

The subjects sat in an electrically shielded, partially soundproofed cubicle, into which a sufficient level of white noise was presented to mask extraneous noises. The surface EEG was derived from a 9-mm Grass gold-cup scalp electrode placed $2.5 \mathrm{~cm}$ above the inion on the midline $(\mathrm{Oz})$ referenced to the right earlobe (A2). EEGs were amplified with a Grass $7 \mathrm{WC}$ ac polygraph with high- and low-frequency filters set at 35 and $1 \mathrm{~Hz}$, respectively. A DataCom signal-averaging system was used to average VEPs for $300 \mathrm{msec}$ after each light flash (dwell time of $4.7 \mathrm{msec}$ ). All VEPs were the average of 64 individual responses. The identification and quantification of the VEP measures was performed by the minicomputer.

\section{RESULTS}

To identify those latencies of the VEP most sensitive to the nature of pattern in the light flashes, a variance analysis procedure was employed (Musso \& Harter, 1975; Towle \& Harter, 1977). In this procedure, the standard deviation of the amplitude of the eight VEPs to the eight different patterns was obtained at each latency of the VEP for each subject. This procedure revealed that the variation in average VEP amplitude, due to changes in check size, was greatest at two points in time after the light flash: one between 115 and $140 \mathrm{msec}(\mathrm{N} 120)$ and the other between 185 and $240 \mathrm{msec}$ (P220), depending on the subject. Examples of these changes are illustrated for three subjects in Figure 2A. The peak of the variance curves defined the latency at which VEP amplitude was measured in reference to the average voltage of the first $50 \mathrm{msec}$ after the flash. If the subject's acuity was so poor that no peak appeared in the standard deviation function (i.e., all eight VEPs were the same), the amplitude measures were taken at the average latency of the other subjects.

Preliminary inspection of the data indicated that the slopes of the small check size portion of the VEP amplitude functions were related to visual acuity - the better the acuity, the greater the effect of check size on VEP amplitude and the greater the slope. Jaeger acuity was therefore quantitatively pre-
$A$
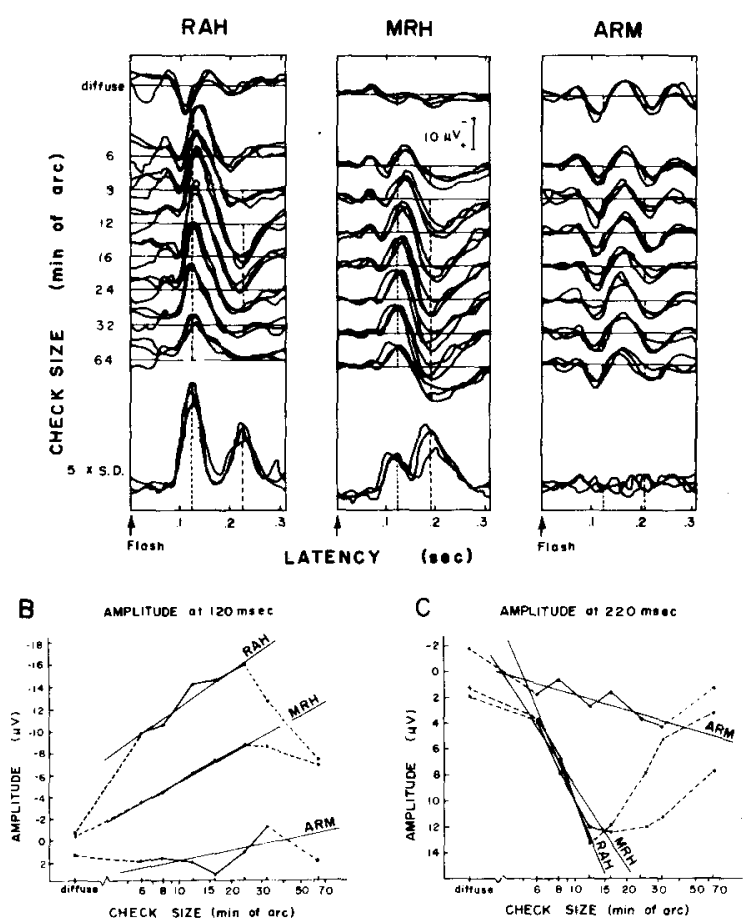

Figure 2. (A) Evoked potentials to diffuse and checkerboard flashes from representative subjects with Jaeger recognition visual acuities of 2.2 (R.A.H.), 1.1 (M.R.H.), and .5 (A.R.M.). Lowest tracings represent variance analyses, the peaks of which define the maximum effect of check size and the latencies at which amplitude measures were taken (N120 and P220, see test). Dotted lines indicate amplitude from baseline. (B) Amplitude of N120 plotted as a function of $\log$ check size for same three subjects. Regression lines were best-fit to the descending portion of the VEP amplitude function (solid lines). The slope of these regression lines was used to predict perceptual visual acuity. (C) Same as "B" but for the P220 measure of VEP amplitude. Note that a decrease in amplitude is indicated by a downward deflection in " $B$ " and an upward deflection in " $C$," since " $B$ " and " $C$ " reflect negative and positive components, respectively.

dicted from the slope of the function between VEP amplitude (microvolts) and check size (log minutes) in the following manner. The least-squares method was used to best-fit regression lines to the descending, small check size portion of the size-amplitude functions, as illustrated in Figure $2 \mathrm{~B}$ and $2 \mathrm{C}$ (solid lines) for the N120 and P220 measures, respectively. Then the correlations between the Jaeger acuity scores of the 11 subjects and the slope of N120 and P220 measures were computed $(r=-.80, p$ $<.003$, and $r=.66, p<.03$, respectively). Finally, regression Equations 1 and 2 (Table 1) were used as a basis for predicted Jaeger acuity on the basis of the slopes of the $\mathrm{N} 120$ and $\mathrm{P} 220$ functions, respectively (Table 1).

When the slopes from both $\mathrm{N} 120$ and P220 were used to predict Jaeger acuity scores using multiple 
regression (Equation 3), $74 \%$ of the variance in Jaeger acuity scores could be accounted for $(r=$ $.86, \mathrm{p}<.005$, df $=2,8$ ). This relationship between the observed and predicted Jaeger acuity scores is illustrated in Figure 3. For example, subject R.A.H. had a predicted Jaeger acuity score of $\mathbf{2 . 1}$ from Equation 3, as compared to an observed score of 2.2. The standard error of the estimate for predicting Jaeger acuity on the basis of Equation 3 was .42 .

\section{DISCUSSION}

The results of the present study corroborate previous studies which indicate that perceptual acuity can be estimated completely objectively from visual evoked potentials-that is, without a behavioral response from the subject or interpretive observations from the experimenter (Grall et al., 1976; Towle \& Harter, 1977). The highest correlation was a multiple correlation taking into account both VEP measures. The increased predictive ability of the multiple correlation implies that the N120 and P220 measures of VEP amplitude vary somewhat independently and may, therefore, reflect different aspects of pattern information processing.

These results extend previous studies of objective methods of estimating adult perceptual visual acuity. Reinecke and Cogan (1958) reported a correlation of $.664\left(r^{2}=.44\right)$ between Snellen and an optokinetic nystagmus measures of visual acuity. The predictive ability reported here between Jaeger acuity scores and the evoked potential was substantially higher $\left(r^{2}=.74\right)$.

Sufficient data are not yet available for determining which of the four strategies portrayed in Figure 1 is the most accurate predictor of perceptual visual acuity. Only the present investigation and one previous study (Towle \& Harter, 1977) have quantitatively related VEP and perceptual measures of visual acuity. The predictive ability of the slope procedure employed in the present study is comparable to that of the pattern threshold procedure employed in the previous study $\left(r-.86, r^{2}-.74\right)$. These two studies were comparable in terms of the range of the subjects' visual acuities and the psychophysical measures of acuity employed.

One difficulty in directly comparing the different statistical procedures by performing them on the

Table 1

Regression liquations Used to Predict Jaeger Acuity on the Basis of the Visual livoked Potential

$\begin{array}{lll}\text { Jacger } & 436,105 \text { (Slope N } 12(1)^{*} & \text { (1) } \\ \text { Jacger } & 712+045 \text { (Slope P2201) } & \text { (2) } \\ \text { Jacper } & 320 \text { (182 (Slop: N1201) } 1124 \text { (Slope P220) } & \text { (1) }\end{array}$

*iser $|e x|$

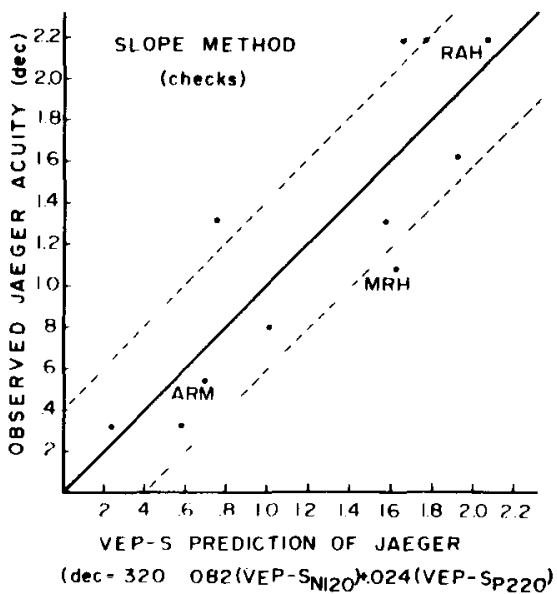

Figure 3. Scatter diagram of observed Jaeger acuity scores and Jaeger acuity predicted on the basis of the VEP when both N120 and P220 were used as predictors in a multiple regression equation (Fquation 3, Tahle 1). Solid line indicates perfect prediction. Dotted lines indicate standard error of the estimate $(68 \%$ confidence interval).

same data is that different ranges of pattern element sizes are required in order to obtain data applicable to each procedure. The stimulus element sizes required to determine the slope of the VEP amplitude function in the present experiment (6 to $64 \mathrm{~min}$ ) were different from those required to determine VEP pattern threshold in the previous experiment (.75 to $19 \mathrm{~min})$. A sufficient range $(.75$ to $180 \mathrm{~min})$ and number of element sizes (at least 15) have not been employed in the same study to directly compare the accuracy of the different statistical procedures. This would allow the use of multiple correlations, which, no doubt, would improve the accuracy of VEP estimates of perceptual acuity beyond those obtained in this and previous studies.

\section{REFERENCES}

Hrkkı., M. A., \& WATkins, D. W. Cirating resolution and refraction in the cal estimated from evoked cerebral potentials. Vision Resparch, 1973, 13, 403-413.

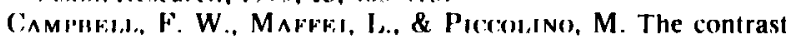
sensitivity of the cal. Journal of Phvsiology, 1973, 229, 719-731.

FurF, $A$ N, I). N., \& MAn(i, E. Vistual acuity development coincides with the sensitive period in kittens Nature, 1975, 254. 614.615 .

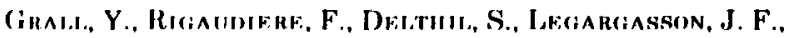
\& Sominil,t.F. J. Potentiels évoqués et acuité visuelle. Vision Research, 1976. 16, 1007-1012.

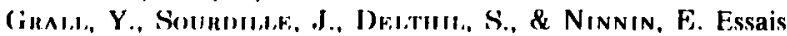
de mesure de l'acuité visuelle chez l'enfant par des methodes ohjectives: l.e nystagmus et les potentiels évoqués. Bulletin et Mémories de la Sociéte Française d'Ophralmologie (Paris), $1973.86,56.59$.

HARTF, M. R., DFATON, F. K.. \& OMOM. J. V. Pattern visual evoked potentials in infants. In J. Fi. Desmedt (Ed.), Visual evoked motentials in man: New developments. Oxford: Clarendon Press. 1977 (a)

HАRTF, M. R., DFATON, F K., \& OIWM. J. V. Maturation of visual preference and evoked potentials in 6-45 day old infants: 
Effects of check size, visual acuity, and refractive error. Journal of Electroencephalography and Clinical Neurophysiology, 1977, 42, 595-607. (b)

Harter, M. R., \& SuitT, C. D. Visually-evoked cortical responses and pattern vision in the infant: A longitudinal study. Psychonomic Science, 1970, 18, 235-237.

HaRTER, M. R., \& White, C. T. Evoked cortical responses to checkerboard patterns: Effect of check-size as a function of visual acuity. Electroencephalography and Clinical Neurophysiology, 1970, 28, 48-54.

Marg, E., Freeman, D. N., Peltzman, P., \& Goldstein, P. J. Visual acuity development in human infants: Evoked potential measurements. Investigative Ophthalmology, 1976, 15, 150-153.

Musso, M. F., \& Harter, M. R. Visually evoked potentials and selective masking with patterned flashes of different spatial frequencies. Vision Research, 1975, 15, 231-238.

REinecke, R. D., \& Cogan, D. G. Standardization of objective visual acuity measurements. Archives of Ophthalmology, 1958, 60, 418-421.
Reitveld, W. J., Tordoir, W. E. M., Hagenouw, J. R. B., Lubbers, J. A., \& Spoor, A. C. Visual evoked responses to blank and to checkerboard patterned flashes. Acta Physiologica et Pharmacologica Neerlandica, 1967, 14, 259-285.

Soкol, S., \& Doвson, V. Pattern reversal visually evoked potentials in infants. Investigative Ophthalmology, 1976, 15, 58-62.

TOWLE, V. L., \& HARTER, M. R. Objective determination of human visual acuity: Pattern evoked potentials. Investigative Ophthalmology and Visual Science, 1977, 16, 1073-1076.

\section{NOTE}

1. The metric value for each Jaeger type size indicates the maximum distance for which a person with 20/20 Snellen acuity can read the line. Decimal visual acuity was estimated from the Jaeger card by the formula: decimal visual acuity $=$ metric value $/ .81$.

(Received for publication December 7, 1978; revision accepted February 5, 1979.) 